\title{
Mott transition, spin-orbit effects, and magnetism in $\mathrm{Ca}_{2} \mathrm{RuO}_{4}$
}

\author{
Guoren Zhang ${ }^{1}$ and Eva Pavarini ${ }^{1,2}$ \\ ${ }^{1}$ Institute for Advanced Simulation, Forschungszentrum Jülich, D-52425 Jülich, Germany \\ ${ }^{2}$ JARA High-Performance Computing, RWTH Aachen University, 52062 Aachen, Germany \\ (Received 23 November 2016; revised manuscript received 26 January 2017; published 23 February 2017)

\begin{abstract}
In this work, we study the effects of spin-orbit and Coulomb anisotropy on the electronic and magnetic properties of the Mott insulator $\mathrm{Ca}_{2} \mathrm{RuO}_{4}$. We use the local-density approximation + dynamical mean-field approach and spin-wave theory. We show that, contrary to a recent proposal, the Mott metal-insulator transition is not induced by the spin-orbit interaction. We confirm that, instead, it is mainly driven by the change in structure from long to short $\mathbf{c}$-axis layered perovskite. We show that the magnetic ordering and the anisotropic Coulomb interactions play a small role in determining the the size of the gap. The spin-orbit interaction turns out to be essential for describing the magnetic properties. It not only results in a spin-wave gap, but it also enlarges significantly the magnon bandwidth.
\end{abstract}

DOI: 10.1103/PhysRevB.95.075145

\section{INTRODUCTION}

The $4 d^{4}$ layered perovskite $\mathrm{Ca}_{2} \mathrm{RuO}_{4}$ (Fig. 1) is made of planes of corner-sharing $\mathrm{RuO}_{6}$ octahedra. It belongs to the $\mathrm{Ca}_{2-x} \mathrm{Sr}_{x} \mathrm{RuO}_{4}$ family, well known for its exotic electronic properties, including, among others, spin-triplet superconductivity [1], Hund's coupling physics [2,3], heavy-fermion [4] and spin-glass behavior [5], as well as a series of structural, magnetic, and electronic phase transitions [6,7]. $\mathrm{Ca}_{2} \mathrm{RuO}_{4}$ itself exhibits a peculiar paramagnetic metal-insulator transition [6] (MIT) at $T_{\mathrm{MIT}}=360 \mathrm{~K}$, basically concurrent with the change from L-Pbca (long $\mathbf{c}$ axis) to $\mathrm{S}-\mathrm{Pbca}$ (short $\mathbf{c}$ axis) structure $[8,9]$ at $T_{\mathrm{S}}=356 \mathrm{~K}$. Similar transitions have been reported when $\mathrm{Ca}$ is partially replaced by $\mathrm{Sr}(x \leqslant 0.2)$ or under pressure [10,11]. The origin of the MIT has been intensively investigated, both experimentally [5-15] and theoretically [16-26]. Electronically, $\mathrm{Ca}_{2} \mathrm{RuO}_{4}$ is characterized by $2 / 3-$ filled $t_{2 g}$ bands $\left(t_{2 g}^{4} e_{g}^{0}\right.$ electronic configuration). Because of the layered structure, the ratio between the $x z / y z$ and $x y$ bandwidth, $R=W_{x z / y z} / W_{x y}$, takes the value $R \sim 0.5$. There is a general agreement that the MIT is caused by strong correlations, i.e., by the screened Coulomb interaction tensor part of the Hamiltonian. Its actual nature has been hotly debated, however. Early on, an orbital-selective Mott transition (OSMT) scenario [24] was proposed in which the orbital polarization $p=n_{x y}-\left(n_{x z}+n_{y z}\right) / 2$ changes from the value $p=1$ below the transition ( $x y$ orbital order, $n_{x y}=2, n_{x z}+n_{y z}=2$ ) to $p=-1 / 2\left(n_{x y}=1, n_{x z}+n_{y z}=3\right)$ in the metallic phase. An alternative proposal was a single Mott transition [17], assisted, however, by the tetragonal crystal-field splitting [27], $\varepsilon_{\mathrm{CF}}=\varepsilon_{x z / y z}-\varepsilon_{x y}>0$. From angle-resolved photoemission (ARPES) data, for $x=0.2$ a new type of OSMT ( $p=$ $1 / 4, n_{x y}=1.5$ ) was inferred [13]; other ARPES experiments reported, however, three metallic bands [14] and no OSMT. Later, accurate local-density approximation + dynamical mean-field theory (LDA+DMFT) calculations [23] showed that the change in crystal structure from L-Pbca to S-Pbca is a decisive factor, leading to a reduction of the bandwidth ratio $R$ and to an enhancement of the crystal-field splitting $\varepsilon_{\mathrm{CF}}$; in the metallic L-Pbca phase, the orbital polarization is $p \sim 0$ (no orbital order) and three metallic bands are obtained, in line with ARPES results from Ref. [14]. Very recent ARPES data for the insulating phase of $\mathrm{Ca}_{2} \mathrm{RuO}_{4}$ appear also in line with LDA+DMFT calculations [3].

Recently, however, these conclusions were challenged by a LDA+U study, which proposes a different scenario [22]. In the latter, it is the Coulomb-enhanced spin-orbit interaction, neglected in LDA+DMFT studies of the MIT so far, to actually induce the transition. Indeed, several works point to a relevant role of the spin-orbit coupling for the electronic structure of layered ruthenates [28-32]. To further complicate the picture, it was shown recently that, surprisingly, the effects of anisotropic Coulomb interactions-i.e., the terms with symmetry lower than $\mathrm{O}(3)$ - are crucial to reproduce the experimental Fermi surface of $\mathrm{Sr}_{2} \mathrm{RuO}_{4}$ [30]. This suggests that they could also play an important role in the metal-insulator transition of $\mathrm{Ca}_{2} \mathrm{RuO}_{4}$, perhaps changing the actual form of the ground state. It becomes necessary, therefore, to systematically reanalyze the metal-insulator transition, explicitly accounting for the effects of both spin-orbit interaction and Coulomb anisotropy.

Remarkably, even for the magnetic phase the role of the spin-orbit interaction is unclear [33-36]. Once more, competing scenarios have been proposed. In the first, the spinorbit interaction can be treated as a perturbation modifying the spin Hamiltonian for the Hund's rule $S=1$ ground multiplet [33,34]; in this picture, the magnetic interactions are described via an $S=1$ Heisenberg-like magnetic-exchange model-both an isotropic [33] and a strongly anisotropic Heisenberg Hamiltonian [34] have been put forward-plus a spin-orbit-induced single-ion anisotropy term, described via a tensor $D$. In the second scenario, the spin-orbit interaction leads to the formation of a zero total angular momentum state $[35,36]\left(j_{t}=0\right)$, and magnetism is therefore of the Van Vleck type.

In this work, we analyze the problem by using the LDA+DMFT approach including explicitly the spin-orbit interaction and the anisotropic Coulomb interaction. The paper is organized as follows. In Sec. I, we describe the model and method used. In Sec. II, we present and analyze the results in the paramagnetic phase. We show that the spin-orbit interaction reduces the gap, contrary to the conclusions from static mean-field theory. We also show that the effect is small, 


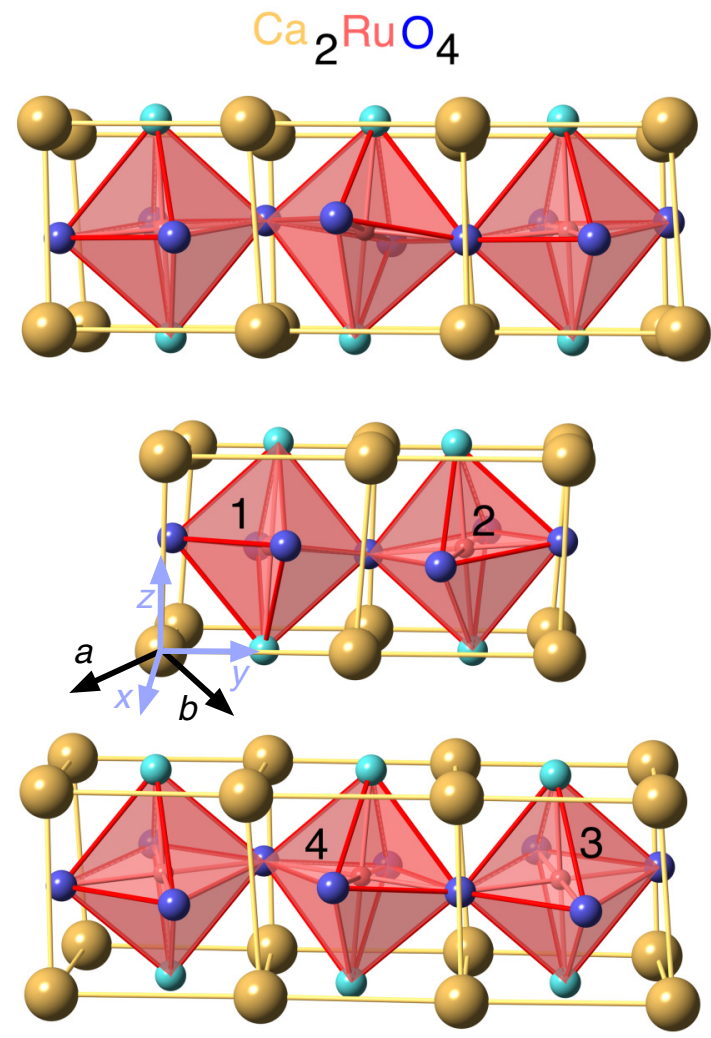

FIG. 1. The S-Pbca structure [8,9] of $\mathrm{Ca}_{2} \mathrm{RuO}_{4}$. The pseudotetragonal axes are $\mathbf{x} \sim(\mathbf{a}+\mathbf{b}) / 2, \mathbf{y} \sim(\mathbf{b}-\mathbf{a}) / 2$, and $\mathbf{z}=\mathbf{c}$. Ru sites $i=2,3,4$ transform into the equivalent site $i=1$ via the symmetry operations $\mathbf{a} \rightarrow-\mathbf{a}(i=2), \mathbf{b} \rightarrow-\mathbf{b}(i=3)$, and $\mathbf{c} \rightarrow-\mathbf{c}(i=4)$.

and we confirm that the MIT is indeed mostly driven by the L-Pbca $\rightarrow$ S-Pbca change in structure. In Sec. III, we discuss the magnetic interaction, and we calculate, using spin-wave theory, the magnon spectrum. We show that the insulating ground state is not the $j_{t}=0$ state. We show that the spin-orbit-induced single-ion anisotropy is essential to explain the spin-wave spectrum. Finally, we give our conclusion in Sec. IV.

\section{MODEL AND METHOD}

We use the local-density-approximation+dynamical meanfield theory approach augmented with spin-wave theory. We first perform LDA calculations [37] with and without spinorbit coupling using the full-potential linearized augmented plane-wave method, as implemented in WIEN2K code [38]. Next we construct $t_{2 g}$ Wannier functions via projectors and a maximal localization procedure [39]. Finally, we build the Hubbard model for the low-lying $t_{2 g}$ states,

$$
\begin{aligned}
H= & -\sum_{i i^{\prime}} \sum_{m m^{\prime}} \sum_{\sigma \sigma^{\prime}} t_{m \sigma, m^{\prime} \sigma^{\prime}}^{i, i_{i m \sigma}^{\prime}} c_{i^{\prime} m^{\prime} \sigma^{\prime}}^{\dagger} \\
& +\frac{1}{2} \sum_{i} \sum_{m m^{\prime} p p^{\prime}} \sum_{\sigma \sigma^{\prime}} U_{m m^{\prime} p p^{\prime}} c_{i m \sigma}^{\dagger} c_{i m^{\prime} \sigma^{\prime}}^{\dagger} c_{i p^{\prime} \sigma^{\prime}} c_{i p \sigma} \\
& -H_{\mathrm{dc}} .
\end{aligned}
$$

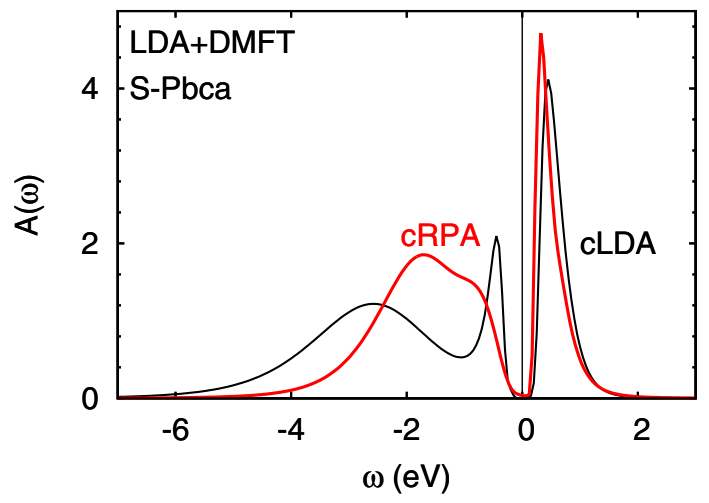

FIG. 2. Total LDA+DMFT spectral function at $T=200 \mathrm{~K}$ for the S-Pbca structure. Light lines (cRPA): $U=2.3 \mathrm{eV}$ and $J=0.4 \mathrm{eV}$. Dark lines (cLDA): $U=3.1 \mathrm{eV}$ and $J=0.7 \mathrm{eV}$.

Here, $c_{i m \sigma}^{\dagger}\left(c_{i m \sigma}\right)$ is the creation (annihilation) operator for the Wannier state with orbital quantum number $m=x y, y z, x z$ and spin $\sigma$ at site $i$, and $n_{i m \sigma}=c_{i m \sigma}^{\dagger} c_{i m \sigma}$. The terms $t_{m \sigma, m^{\prime} \sigma^{\prime}}^{i, i^{\prime}}$ yield for $i=i^{\prime}$ the on-site energies $\varepsilon_{m \sigma, m^{\prime} \sigma^{\prime}}=-t_{m \sigma, m^{\prime} \sigma^{\prime}}^{i, i}$ and for $i \neq$ $i^{\prime}$ the hopping integrals. The parameters $U_{m m^{\prime} n n^{\prime}}$ are elements of the screened Coulomb interaction tensor. For $t_{2 g}$ states in a free atom, the essential terms are [40] the direct Coulomb interaction, $U_{m m^{\prime} m m^{\prime}}=U_{m, m^{\prime}}=U-2 J\left(1-\delta_{m, m^{\prime}}\right)$, the exchange Coulomb interaction, $U_{m m^{\prime} m^{\prime} m}=J$, the pairhopping term, $U_{m m m^{\prime} m^{\prime}}=J$, and the spin-flip term, $U_{m m^{\prime} m^{\prime} m}=$ $J$. For $\mathrm{Ca}_{2} \mathrm{RuO}_{4}$, the site symmetry of Ru sites is only $C_{i}$. The main anisotropy effects are given by the two tetragonal terms $\Delta U=U_{x z, x z}-U_{x y, x y}$ and $\Delta U^{\prime}=U_{x z, y z}-U_{x y, y z}$; we therefore study the effects of those terms in particular.

We perform calculations with two sets of screened parameters. The first, $U=3.1 \mathrm{eV}$ and $J=0.7 \mathrm{eV}$, has been obtained for $\mathrm{Sr}_{2} \mathrm{RuO}_{4}$, the sister compound of $\mathrm{Ca}_{2} \mathrm{RuO}_{4}$, via the constrained local-density approximation (cLDA) [41]. These parameters have been already successfully used [23] to describe the metal-insulator transition in $\mathrm{Ca}_{2} \mathrm{RuO}_{4}$ with the LDA+DMFT approach. The second set of parameters are the constrained random-phase approximation (cRPA) values [42], $U=2.3 \mathrm{eV}$ and $J=0.4 \mathrm{eV}$. Remarkably, both sets yield an insulating solution with $x y$-like orbital order for the S-Pbca structure. The comparison between LDA+DMFT spectra for the cLDA and the cRPA parameter sets is shown in Fig. 2. The main differences between the two results are (i) the size of the gap (slightly smaller for the cRPA values) and (ii) the shape of the lower Hubbard band. Both spectral functions exhibit Hubbard bands more or less in line with the spectra measured in photoemission, $\mathrm{x}$-ray fluorescence emission, and $\mathrm{X}$-ray absorption spectroscopy experiments [41,43-45], thus it is difficult, based on experiments available so far, to decide conclusively in favor of one set or the other. For this reason we will present, when necessary, results for both the cLDA and cRPA parameter sets. Finally, $H_{\mathrm{dc}}$ is the double-counting correction [46]. For what concerns the spin-orbit interaction, which is by construction included in the Hamiltonian in $\mathrm{LDA}+\mathrm{SO}$ calculations, the on-site term can be written as

$$
H_{\mathrm{SO}}=\sum_{i \mu} H_{\mathrm{SO}}^{i \mu}=\sum_{i \mu} \sum_{m \sigma m^{\prime} \sigma^{\prime}} \lambda_{\mu}^{i} \xi_{m \sigma m^{\prime} \sigma^{\prime}}^{i \mu} c_{i m \sigma}^{\dagger} c_{i m^{\prime} \sigma^{\prime}},
$$


TABLE I. Phase transformation for the different crystal-field orbitals used in LDA+SO+DMFT calculations, with $\phi_{L}=\pi / 2$, $\phi_{S}=-\pi / 4$, and $\theta_{i \downarrow}=-\theta_{i \uparrow}$.

\begin{tabular}{llll}
\hline \hline & $e^{i \theta_{1 \uparrow}}$ & $e^{i \theta_{2 \uparrow}}$ & $e^{i \theta_{3 \uparrow}}$ \\
\hline $\operatorname{L-Pbca}(400 \mathrm{~K})$ & $e^{i \phi_{L}}$ & $e^{i \phi_{L}-i 0.239 \pi}$ & $e^{i \phi_{L}+i 0.262 \pi}$ \\
$\operatorname{S-Pbca}(180 \mathrm{~K})$ & $e^{i \phi_{S}}$ & $e^{i \phi_{S}-i 0.664 \pi}$ & $e^{i \phi_{S}+i 0.824 \pi}$ \\
\hline \hline
\end{tabular}

where $\xi_{m \sigma m^{\prime} \sigma^{\prime}}^{i \mu}=\left\langle m \sigma\left|s_{\mu}^{i} l_{\mu}^{i}\right| m^{\prime} \sigma^{\prime}\right\rangle$ and $\mu$ labels the pseudocubic axes. We extract the coupling $\lambda_{\mu}^{i}=\lambda_{\mu}$ by comparing the LDA and LDA+SO Hamiltonian and find the value $\lambda_{z} \sim \lambda_{x y}=\lambda \sim 106 \mathrm{meV}$ for both the L-Pbca and S-Pbca structures. This value is similar to the one we previously obtained [30] for $\mathrm{Sr}_{2} \mathrm{RuO}_{4}$. The LDA tetragonal splitting $\delta \lambda=\lambda_{x y}-\lambda_{z}$ is negligible. The effect of the spin-orbit on the hopping integrals is also small.

To deal with many-body Hamiltonians of arbitrary symmetry, we have developed generalized LDA+DMFT solvers $[23,30,47]$ based on the continuous-time (CT) quantum Monte Carlo (QMC) [48] technique. Here, we use the interaction-expansion [49] flavor (CT-INT) of this solver [23], which we recently extended to include the spin-orbit interaction as well [30]. In the calculation with spin-orbit coupling, we work with a full $6 \times 6$ self-energy matrix $\Sigma_{m \sigma, m^{\prime} \sigma^{\prime}}$ in spin-orbital space; furthermore, in order to reduce the sign problem, we change the basis via a unitary transformation $\left|m_{c}\right\rangle_{\sigma}=\hat{U}|m\rangle_{\sigma}$ that minimizes the imaginary part of the $G(\tau)$ Green-function matrix, where $\tau$ is the imaginary time. For $\mathrm{Ca}_{2} \mathrm{RuO}_{4}$ the matrix $\hat{U}$ can be written as $\hat{U}=e^{i \hat{\theta}} \hat{B}$, where $\hat{B}$ is the real, spin-independent, matrix that diagonalizes the LDA on-site energy matrix $\varepsilon_{m \sigma, m^{\prime} \sigma}$, yielding the LDA crystal-field energies $\varepsilon_{m_{c}}$, and $\hat{\theta}$ is a matrix of phases, diagonal in spin and orbitals. The elements of the matrix $\hat{\theta}$ can be read in Table I. After this transformation, the local Hamiltonian becomes real apart from small imaginary corrections $<2 \mathrm{meV}$; the imaginary part of $G(\tau)$ is also tiny and can be neglected in the calculation. Finally, we have previously shown that spin-flip and pair-hopping terms have only small effects on the LDA+DMFT spectral function for $\mathrm{Ca}_{2} \mathrm{RuO}_{4}$ [23]; thus, in most calculations presented here, we neglect these terms to speed up the calculations. In representative cases, we explicitly check that this approximation does not affect the conclusions. Finally, for the analytic continuation of imaginary-time data to the real frequency axis, we adopt the maximum-entropy approach [50].

\section{RESULTS AND DISCUSSION}

\section{A. Metal-insulator transition}

In this section, we discuss the effects of spin-orbit interaction and Coulomb anisotropy on the metal-insulator transition.

\section{Spin-orbit effects}

Let us call $\left|m_{c}\right\rangle_{\sigma}=\hat{B}|m\rangle_{\sigma}$ the crystal-field states in the absence of spin-orbit interaction. For the L-Pbca structure

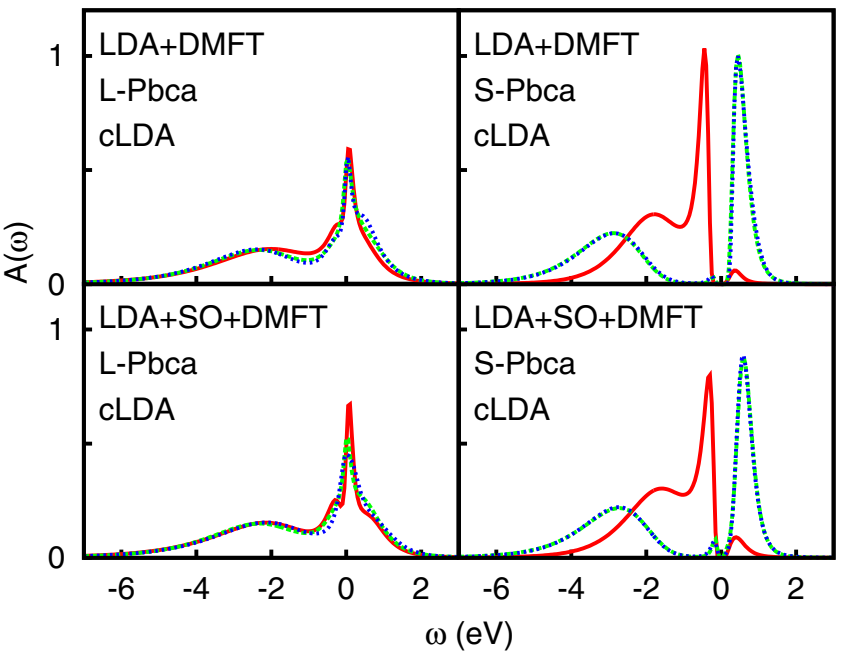

FIG. 3. LDA+DMFT (upper panels) and LDA+SO+DMFT (lower panels) $t_{2 g}$ spectral-function matrix. Solid lines: $A_{1,1}$. Dashed and dotted lines: $A_{2,2}$ and $A_{3,3}$. Left: L-Pbca, $T=580 \mathrm{~K}$. Right: S-Pbca, $T=290$ K. Calculations performed with cLDA Coulomb parameters.

(400 K), the $\hat{B}$ matrix is given by

$$
\left(\begin{array}{l}
|1\rangle \\
|2\rangle \\
|3\rangle
\end{array}\right)=\left(\begin{array}{rrr}
0.972 & 0.224 & -0.075 \\
0.171 & -0.886 & -0.432 \\
0.163 & -0.407 & 0.899
\end{array}\right)\left(\begin{array}{l}
|x y\rangle \\
|y z\rangle \\
|x z\rangle
\end{array}\right),
$$

while for the S-Pbca structure $(180 \mathrm{~K})$ it is given by

$$
\left(\begin{array}{l}
|1\rangle \\
|2\rangle \\
|3\rangle
\end{array}\right)=\left(\begin{array}{rrr}
0.972 & 0.195 & -0.130 \\
-0.161 & 0.959 & 0.233 \\
0.170 & -0.205 & 0.964
\end{array}\right)\left(\begin{array}{c}
|x y\rangle \\
|y z\rangle \\
|x z\rangle
\end{array}\right) .
$$

The crystal-field splittings $\Delta_{1, m_{c}}=\varepsilon_{m_{c}}-\varepsilon_{1}$ take the values $\Delta_{1,2} \sim 105 \mathrm{meV}$ and $\Delta_{1,3} \sim 116 \mathrm{meV}$ for the L-Pbca structure and $\Delta_{1,2} \sim 309 \mathrm{meV}$ and $\Delta_{1,3} \sim 320 \mathrm{meV}$ for the S-Pbca structure. The differences in crystal-field splittings and states between 11 and $180 \mathrm{~K} \mathrm{S-Pbca}$ structure are negligible. Here and in the following, matrices and states are given for site 1 in Fig. 1. For both structures, the ground state $|1\rangle$ has a dominant $x y$ component, while $|2\rangle$ and $|3\rangle$ are mostly $y z$ - and $x z$-like.

Let us now analyze the LDA+DMFT results. In line with previous LDA+DMFT calculations [23], we find that the L-Pbca structure yields a metallic solution with orbital polarization $p \sim 0$ and the S-Pbca insulating with $p \sim 1$ and $x y$-like orbital order [20,23]. This is shown in Fig. 3. Indeed, the fully occupied natural orbital for cLDA Coulomb parameters is

$$
\left|\alpha_{1}\right\rangle_{\sigma}=0.993|x y\rangle_{\sigma}+0.118|y z\rangle_{\sigma}-0.023|x z\rangle_{\sigma}
$$

with spin degeneracy 2 and $n_{\alpha_{1}}=n_{\alpha_{1 \uparrow}}+n_{\alpha_{1 \downarrow}} \sim 1.95$ at $T=$ $290 \mathrm{~K}$; this state is close to the lowest-energy crystal-field orbital, $\left|\alpha_{1}\right\rangle \sim|1\rangle$. Switching on the spin-orbit coupling 


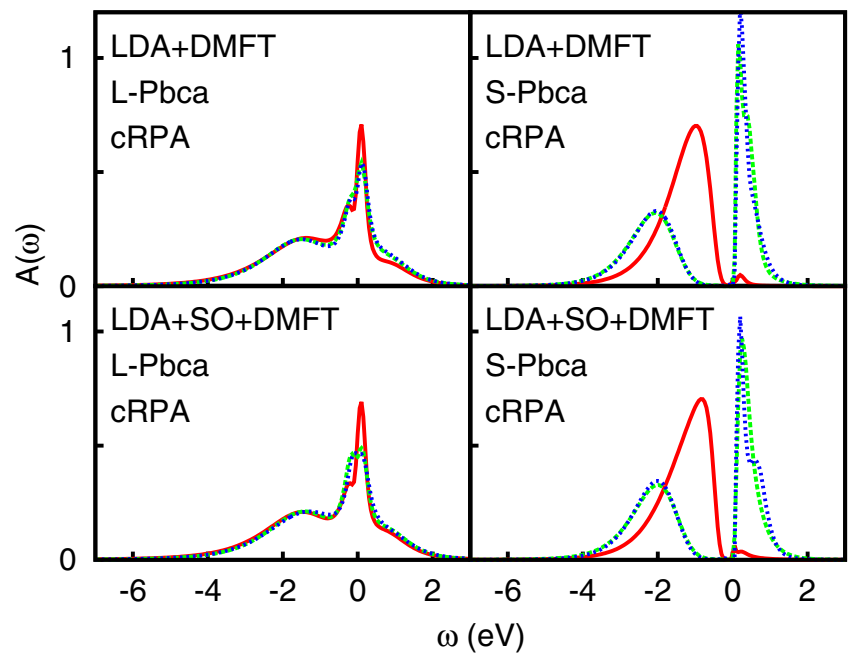

FIG. 4. LDA+DMFT (upper panels) and LDA+SO+DMFT (lower panels) $t_{2 g}$ spectral-function matrix. Solid lines: $A_{1,1}$. Dashed and dotted lines: $A_{2,2}$ and $A_{3,3}$. Left: L-Pbca, $T=580 \mathrm{~K}$. Right: $\mathrm{S}-\mathrm{Pbca}, T=290 \mathrm{~K}$. Calculations performed with cRPA Coulomb parameters.

transforms $\left|\alpha_{1}\right\rangle_{\sigma}$ into the Kramers doublet

$$
\begin{aligned}
\left|\alpha_{1}^{\mathrm{so}}\right\rangle_{\sigma}= & s_{\sigma} 0.972|x y\rangle_{\sigma} \\
& s_{\sigma} 0.191 e^{-i s_{\sigma} 0.03 \pi}|y z\rangle_{\sigma}+0.075 e^{-i s_{\sigma} 0.09 \pi}|y z\rangle_{-\sigma} \\
& -s_{\sigma} 0.085 e^{-i s_{\sigma} 0.09 \pi}|x z\rangle_{\sigma}-0.073 e^{+i s_{\sigma} 0.39 \pi}|x z\rangle_{-\sigma},
\end{aligned}
$$

where $s_{\sigma}=+1$ for $\sigma=\uparrow$ and $s_{\sigma}=-1$ for $\sigma=\downarrow$ and $n_{\alpha_{1}^{\text {so }}}=\sum_{\sigma} n_{\alpha_{1 \sigma}^{\text {so }}} \sim 1.90$ at $T=290 \mathrm{~K}$. Hence, $x y$-orbital order persists even in the presence of spin-orbit coupling; the latter merely reduces somewhat the $x y$ component. The doublet $\left|\alpha_{1}^{\text {so }}\right\rangle_{\sigma}$ is similar to the doublet obtained by diagonalizing the on-site Hamiltonian in the presence of spin-orbit interaction, $\left|1^{\text {so }}\right\rangle$; the latter has a slightly smaller $x y$ component, $\left|\left\langle x y \mid 1^{\text {so }}\right\rangle\right| \sim 0.956$. All these results and conclusions change very little if we use cRPA instead of cLDA Coulomb parameters.

Remarkably, we find that the spin-orbit coupling reduces the gap for the S-Pbca structure, as can be seen in Figs. 3 and 4 , even if such a reduction is very small. This can be qualitatively understood already in the atomic limit (see the Appendix for details). Let us consider at first an idealized $t_{2 g}^{4}$ atom ( $N=4$ electrons). In the presence of $x y$-orbital ordering, for simplicity we can at first consider the $x y^{2}$ states as inert. Then, the atomic gap in the $A_{x z}(\omega)$ and $A_{y z}(\omega)$ spectral functions is given by

$$
E_{\text {gap }}^{x z \rightarrow x z}\left(\lambda_{z}\right)=U+J-\lambda_{z} .
$$

This happens because the relevant $|N+1\rangle$ and $|N-1\rangle$ multiplets split into doublets that differ by $\lambda_{z}$ in energy, while the lowest-energy $|N\rangle$ multiplet is not modified by the spin-orbit interaction at linear order. The conclusion remains approximately true even if we take into account the $x y^{2}$ states explicitly. In the small $\lambda_{x y} / 2 \varepsilon_{\mathrm{CF}}$ limit, the actual atomic gap is given by

$$
E_{\text {gap }}^{x y \rightarrow x z}\left(\lambda_{z}\right) \sim U-3 J+\varepsilon_{\mathrm{CF}}-\lambda_{z} / 2 .
$$

This is the opposite conclusion with respect to what one finds in LDA+U calculations [22] (static mean-field theory), where, in the nonmagnetic case, the gap is actually increasing with the effective spin-orbit coupling [51]. Remarkably, the situation changes drastically in the absence of crystal-field and $x y$-orbital order, i.e., when the $|N\rangle$ atomic states with $n_{x y}=1$ are degenerate with those $n_{x y}=2$ states, as it happens in the absence of a crystal-field splitting. In that case, the $S=1$ multiplet splits and the lowest energy state has total angular momentum $j_{t}=0$. For such a ground state, the spin-orbit coupling increases the gap. It is important to point out that, in LDA+DMFT calculations, the effective crystal-field enhancement, which ultimately leads to orbital order, is dynamical (frequency-dependent), and it is much larger in the low-frequency limit than in the high-frequency (Hartree-Fock) limit. In particular, we find $\Delta \varepsilon_{\mathrm{CF}}(0) \sim 2 \mathrm{eV}$ and $\Delta \varepsilon_{\mathrm{CF}}(\infty)=-0.18 \mathrm{eV}$ for the cLDA Coulomb parameters, and $\Delta \varepsilon_{\mathrm{CF}}(0) \sim 1.5 \mathrm{eV}$ and $\Delta \varepsilon_{\mathrm{CF}}(\infty)=0.14 \mathrm{eV}$ for the cRPA Coulomb parameters. The spin-orbit coupling is also enhanced dynamically, but it remains smaller than the effective crystal-field splitting, and at low frequencies even sizably smaller. In the case of the metallic L-Pbca structure, the crystal-field enhancement is small in both limits, with or without spin-orbit interaction.

\section{Anisotropic Coulomb interaction effects}

Recently, we have shown that the low-symmetry terms in the Coulomb vertex $\Delta U=U_{x z, x z}-U_{x y, x y}$ and $\Delta U^{\prime}=$ $U_{x z, y z}-U_{x y, y z}$ are very important in determining the shape of the Fermi surface of $\mathrm{Sr}_{2} \mathrm{RuO}_{4}$ [30]. Here we want to analyze the effects of $\Delta U$ and $\Delta U^{\prime}$ on the metal-insulator transition in $\mathrm{Ca}_{2} \mathrm{RuO}_{4}$. Remarkably, due to tilting rotation and the remaining deformations of the octahedra, in $\mathrm{Ca}_{2} \mathrm{RuO}_{4}$, different from in $\mathrm{Sr}_{2} \mathrm{RuO}_{4}$, the $x y$ orbitals are longer range than $y z / x z$ states [52]. Thus we expect that, correspondingly, $\Delta U=U_{x y, x y}-U_{x z, x z}$ and $\Delta U^{\prime}=U_{x y, y z}-U_{x z, y z}$ have a negative, rather than a positive, sign. Furthermore, we find that the difference in spread between $x z$ and $y z$, a measure of the orthorhombicity, is small [52], hence we take $U_{x z, x z} \sim U_{y z, y z}$. Material-specific cRPA or cLDA estimates of $\Delta U$ and its sign would of course be very welcome to confirm these conclusions. Figure 5 shows the total spectral functions of S-Pbca for the representative case $\Delta U=3 \Delta U^{\prime}=-0.45 \mathrm{eV}$. The figure shows that the overall effect of the Coulomb anisotropy is small. The anisotropic Coulomb terms slightly modify the shape of the spectral function and the relative occupations, but the gap is barely changed. Let us compare here in detail LDA+DMFT results with the full Coulomb vertex. The fully occupied doublet, which for $\Delta U=\Delta U^{\prime}=0$ is

$$
|\alpha\rangle_{\sigma}=0.929|x y\rangle_{\sigma}+0.271|y z\rangle_{\sigma}-0.254|x z\rangle_{\sigma},
$$

for $\Delta U=3 \Delta U^{\prime}=-0.45 \mathrm{eV}$ becomes

$$
\left|\alpha_{1}\right\rangle_{\sigma}=0.969|x y\rangle_{\sigma}+0.187|y z\rangle_{\sigma}-0.162|x z\rangle_{\sigma} .
$$

Due to the Coulomb anisotropy terms, and in particular $\Delta U<0$, the occupation of the $x y$ orbital increases, but only slightly. These results altogether lead us to the conclusion that Coulomb anisotropy, although very important for the shape of 


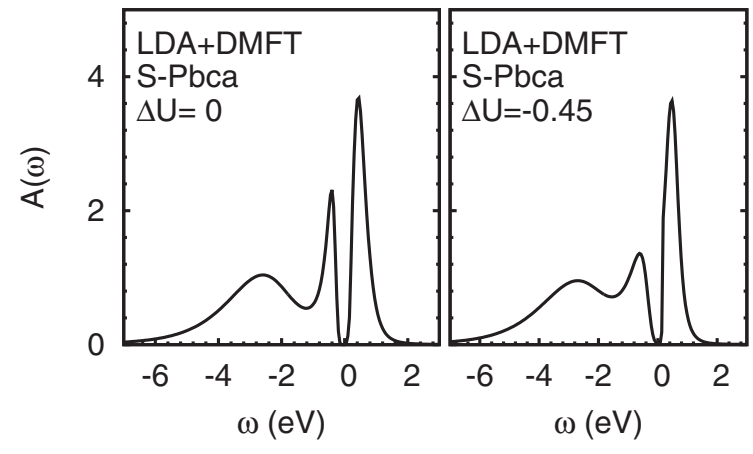

FIG. 5. Total LDA+DMFT $t_{2 g}$ spectral function with anisotropic Coulomb interactions. Left panel: $\Delta U=\Delta U^{\prime}=0$. Right panel: $\Delta U=3 \Delta U^{\prime}=-0.45 \mathrm{eV} . T=290 \mathrm{~K}$. These calculations have been done with the full Coulomb vertex at $T=290 \mathrm{~K}$ and for cLDA parameters.

the Fermi surface of correlated ruthenates, does not play a key role in the metal-insulator transition observed in $\mathrm{Ca}_{2} \mathrm{RuO}_{4}$.

\section{Effects of antiferromagnetic ordering}

$\mathrm{Ca}_{2} \mathrm{RuO}_{4}$ orders antiferromagnetically [9] at $T_{N} \sim 110 \mathrm{~K}$. Here we investigate the effects of magnetic order on the spectral function matrix. LDA+DMFT spin-polarized AFM calculations yield a paramagnetic solution at $290 \mathrm{~K}$. Since LDA+DMFT calculations in the $T \rightarrow 0$ limit are very time-consuming, to obtain a sizable magnetic moment we introduce a staggered magnetic field with the same $\mathbf{q}$ vector of the magnetically ordered structure; this leads to staggered magnetic moments $\mu_{z} \sim 1.96 \mu_{B}$ per Ru at $290 \mathrm{~K}$. Even for such a large local moment, the picture remains the same. We find that the system retains $x y$-like orbital order below the magnetic transition, and the magnetic moment comes mostly for the half-filled natural orbitals $\left|\alpha_{2}\right\rangle$ and $\left|\alpha_{3}\right\rangle$, which are almost completely spin-polarized. For what concerns the spectral function, the main effect is a partial spectral-weight transfer within the Hubbard bands and a somewhat larger gap. The differences are more pronounced for the cRPA Coulomb parameter set, as can be seen in Fig. 6.

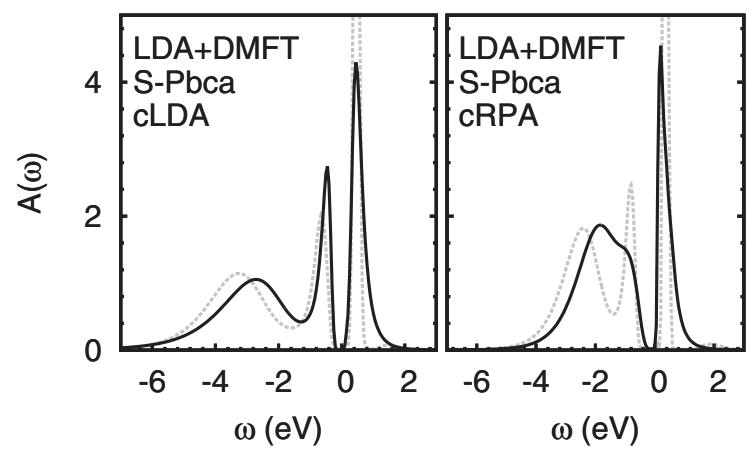

FIG. 6. The LDA+DMFT total $t_{2 g}$ spectral function for the paramagnetic (full line) and antiferromagnetic (dashed line) phase. The AFM spectrum is calculated in the presence of a large staggered antiferromagnetic field yielding a magnetic moment $\mu_{z} \sim 1.96 \mu_{B}$. Both spectra have been calculated at $T=290 \mathrm{~K}$ and for cLDA (left panel) and cRPA (right panel) Coulomb parameters.

\section{B. Low-energy magnetic model and spin waves}

\section{Magnetic Hamiltonian and superexchange couplings}

The spin-orbit coupling has an important role in determining the magnetic structure and magnetic excitations. Two competing models have been proposed so far: in the first scenario, the spin-orbit interaction leads to a total angular momentum $j_{t}=0$ state $[35,36]$; in the second picture, the spin-orbit interaction yields, instead, only perturbative effects (e.g., a magnetic anisotropy tensor) partially removing the degeneracy of the $S=1$ ground-state multiplet [33]. In the first scenario, the magnetic linear-response function is of the Van Vleck type, in the second it arises from the local $S=1$ magnetic moments. Our LDA+SO+DMFT results show that, in the insulating phase, the spin-orbit interaction does not lead to a $j_{t}=0$ ground state [53], which would give $p \sim 0$. Instead, we find a fully occupied Kramers doublet $(p \sim 1)$ with a dominant $x y$ component and a partial (real) mixing to the $x z$ state, as it happens when the crystal field, rather than spin-orbit, determines the ground state. We therefore explore the second picture and calculate the effective spin Hamiltonian in second-order perturbation theory. Toward that end, we assume that the ground-state multiplet is the $S=1$ orbitally ordered state. The resulting $S=1$ generalized Heisenberg-like model takes the form

$$
H_{S}=\frac{1}{2} \sum_{i i^{\prime}} \Gamma^{i, i^{\prime}} \mathbf{S}^{i} \cdot \mathbf{S}^{i^{\prime}}+\sum_{i} \sum_{\mu \mu^{\prime}} D_{\mu \mu^{\prime}}^{i} S_{\mu}^{i} S_{\mu^{\prime}}^{i} .
$$

Here the first term describes the superexchange magnetic interaction, and the second term describes the single-ion anisotropy, while here $\mu, \mu^{\prime}$ are the crystal $\mathbf{a}, \mathbf{b}, \mathbf{c}$ directions. The exchange coupling $\Gamma_{i, i^{\prime}}$ is given by

$$
\begin{aligned}
\Gamma^{i, i^{\prime}} \sim & -\frac{1}{2} \sum_{m_{c}>1}\left[\frac{\left|t_{1, m_{c}}^{i, i \prime^{\prime}}\right|^{2}+\left|t_{m_{c}, 1}^{i, i^{\prime}}\right|^{2}}{U-3 J+\Delta_{1, m_{c}}} \frac{2 J}{U-J+\Delta_{1, m_{c}}}\right] \\
& +\frac{1}{2} \sum_{m_{c}>1} \sum_{m_{c}^{\prime}>1} \frac{\left|t_{m_{c}, m_{c}^{\prime}}^{i, i^{\prime}}\right|^{2}+\left|t_{m_{c}^{\prime}, m_{c}}^{i, i^{\prime}}\right|^{2}}{U+J+\Delta_{m_{c}, m_{c}^{\prime}}} .
\end{aligned}
$$

The LDA crystal-field states $\left|m_{c}\right\rangle_{\sigma}$ are labeled in order of growing on-site energies $\varepsilon_{m_{c}+1} \geqslant \varepsilon_{m_{c}}$. The energy differences are $\Delta_{m_{c}, m_{c}^{\prime}}=\varepsilon_{m_{c}^{\prime}}-\varepsilon_{m_{c}}$, with $\Delta_{1, m_{c}}>0$. We calculate the exchange parameters both for the experimental S-Pbca structure measured at $180 \mathrm{~K}$ and the one measured at $11 \mathrm{~K}$; they yield similar results. In the following, we give the numbers for the $11 \mathrm{~K}$ structure calculated for the cLDA Coulomb parameter sets. For the nearest-neighbor magnetic coupling $\Gamma_{1}=\Gamma^{i, i+(\mathbf{a} \pm \mathbf{b}) / 2}$ we obtain $\Gamma_{1} \sim 2.6 \mathrm{meV}$, basically the same for the bonds along the $\mathbf{x}$ and $\mathbf{y}$ directions. Furthermore, we find that the effects of spin-orbit on the hopping integrals are small, and that the Heisenberg parameters are basically isotropic. The next-nearest-neighbor couplings exhibit instead a small direction dependence, with $\Gamma_{2}^{a}=\Gamma^{i, i+a}=-0.1 \mathrm{meV}$ and $\Gamma_{2}^{b}=\Gamma^{i, i+\mathbf{b}}=0.08 \mathrm{meV}$ along the $\mathbf{b}$ direction, and the interlayer couplings, $\Gamma_{z}^{b}=\Gamma^{i, i+(\mathbf{b}+\mathbf{c}) / 2}=0.30 \mathrm{meV}$ and $\Gamma_{z}^{a}=\Gamma^{i, i+(\mathbf{a}+\mathbf{c}) / 2}=0.54 \mathrm{meV}$. The remaining parameters are tiny and can be neglected. The hopping integrals to the partially occupied orbitals yield a ferromagnetic superexchange contribution, which is responsible for the minus sign in the first 
TABLE II. Single-ion anisotropy tensor $D$ (in meV) for site 1 (see Fig. 1 for definition) for the different structures. These values have been obtained for the cRPA parameters $U=2.3 \mathrm{eV}$ and $J=0.4 \mathrm{eV}$ and isotropic $\lambda_{\text {eff }}=180 \mathrm{meV}$.

\begin{tabular}{|c|c|c|c|c|c|c|c|c|c|}
\hline \multicolumn{4}{|c|}{ L-Pbca $(400 \mathrm{~K})$} & \multicolumn{3}{|c|}{ S-Pbca (180 K) } & \multicolumn{3}{|c|}{ S-Pbca (11 K) } \\
\hline \multirow{3}{*}{$D=$} & $(-41.42$ & 1.82 & $0.00)$ & $(-16.34$ & 0.27 & $0.00)$ & $(-16.00$ & 0.35 & $0.00)$ \\
\hline & 1.82 & -39.63 & 0.00 & 0.27 & -16.40 & 0.00 & 0.35 & -16.24 & 0.00 \\
\hline & 0.00 & 0.00 & $-4.11)$ & 0.00 & 0.00 & $-1.42)$ & 0.00 & 0.00 & $-1.39)$ \\
\hline
\end{tabular}

term of Eq. (6); the latter competes with the remaining antiferromagnetic (positive) contribution and, remarkably, dominates for the coupling $\Gamma_{2}^{a}$. Furthermore, surprisingly, the interlayer exchange couplings $\Gamma_{z}^{a / b}$ are sizably larger than $\Gamma_{2}^{a / b}$, indicating that the system exhibits a small but non-negligible threedimensional character. By using the cRPA parameters, we obtain an overall very similar picture but, as expected, larger couplings, in particular $\Gamma_{1} \sim 5.3 \mathrm{meV}, \Gamma_{2}^{a} \sim-0.02 \mathrm{meV}$, $\Gamma_{2}^{b} \sim 0.18 \mathrm{meV}, \Gamma_{z}^{b} \sim 0.50 \mathrm{meV}$, and $\Gamma_{z}^{a} \sim 0.80 \mathrm{meV}$.

Experimentally, the exchange parameters were estimated via inelastic neutron scattering as $\Gamma_{1} \sim 16 \mathrm{meV}$ in Ref. [33] and $\Gamma_{1} \sim 5.6 \mathrm{meV}$ in Ref. [34]. Our result $\Gamma_{1} \sim 3-6 \mathrm{meV}$ is closer to the second value. The apparent discrepancy with the first work can be ascribed to the spin model used to fit spinwave data, in which only a small in-plane single-ion anisotropy term $D_{b b}$ is included. By using our exchange couplings and a realistic single-ion magnetic anisotropy, we find spin-wave spectra in line with experiments (see the next subsection). The single-ion magnetic anisotropy tensor is given by

$$
D_{\mu \mu^{\prime}}^{i} \sim \sum_{N} \frac{\left\langle 0\left|H_{\mathrm{SO}}^{i \mu}\right| N\right\rangle\left\langle N\left|H_{\mathrm{SO}}^{i \mu^{\prime}}\right| 0\right\rangle}{E_{0}-E_{N}},
$$

where $\{|N\rangle\}$ are the excited atomic multiplet levels and $\left\{E_{N}\right\}$ are their energies, and $|0\rangle$ is the $S=1$ ground-state triplet with energy $E_{0} \sim 2 \varepsilon_{\mathrm{CF}}+6 U-13 J$. Remarkably, our LDA+DMFT calculations yield a sizable enhancement of the spin-orbit couplings. This can be shown by analyzing the offdiagonal elements of the self-energy matrix. The enhancement is dynamical and anisotropic; for the cLDA parameters, the Hartree-Fock contributions (large Matsubara frequency limit) of the enhancement, $\Delta \lambda_{z} \sim-2(U-3 J) n_{2 \uparrow, 3 \uparrow}$ and $\Delta \lambda_{x y} \sim$ $2(U-3 J) n_{1 \uparrow, 3 \downarrow}$, are 30 and $60 \mathrm{meV}$, respectively, while in the low-frequency limit it is about $100 \mathrm{meV}$ at $290 \mathrm{~K}$ for both tensor components. It is important to point out that although this enhancement is large, the effective coupling is sizably smaller than the $900 \mathrm{meV}$ estimated via the static mean field $(\mathrm{LDA}+\mathrm{U})$ in Ref. [22]; this is partly due to the form of the Coulomb interaction used in that work, which differs from the actual atomic Coulomb vertex [54]. A uniform increase of the average coupling $\lambda_{\text {eff }}=\left(\lambda_{x z}+2 \lambda_{x y}\right) / 3$ enhances the strength of the single-ion anisotropy; the anisotropy, $\delta \lambda=$ $\lambda_{x y}-\lambda_{z}>0$, with everything else remaining the same, enhances $D_{a a}$ and $D_{b b}$ with respect to $D_{c c}$.

In Table II we show the single-ion anisotropy tensor for an isotropic $\lambda_{\text {eff }} \sim 180 \mathrm{meV}$ and cRPA Coulomb parameters. For all structures considered here, we find that $D_{a a} \approx D_{b b}<0$ and is much larger in absolute value than $D_{c c}<0$. This indicates that the easy axis is within the $\mathrm{Ru}-\mathrm{O}$ plane, in line with neutron scattering experiments $[9,55]$, and the magnetic anisotropy is to a large extent tetragonal. Remarkably, the $D_{a a} \sim D_{b b}$ element is about twice as large (in absolute value) in the L-Pbca than in the S-Pbca structure; this happens because the $\mathrm{S}$-Pbca structure yields larger tetragonal crystal-field splitting. In our calculations, the easy axis direction rotates from $\hat{\mathbf{e}} \sim-0.85 \hat{\mathbf{a}}+0.53 \hat{\mathbf{b}}$ at $400 \mathrm{~K}$ to $\hat{\mathbf{e}} \sim-0.6 \hat{\mathbf{a}}+0.8 \hat{\mathbf{b}}$ at $11 \mathrm{~K}$, i.e., it rotates progressively toward the $\mathbf{b}$ direction by increasing the distortions. It is worth noticing, however, that within the ab plane, the calculated easy magnetization axis direction depends strongly on the tiny off-diagonal tensor elements $D_{a b}$ and the tiny in-plane anisotropy $D_{a a}-D_{b b}$, and therefore it is very sensitive to experimental errors in the determination of the small orthorhombic distortions. More specifically, we find that the difference in single-ion anisotropy energy gain between $\hat{\mathbf{e}}$ and any other direction in the ab plane is smaller than $1 \mathrm{meV}$, hence it is comparable to errors arising from experimental incertitude on atomic positions or to small neglected magnetic interaction terms. Neutron scattering experiments [9] indicate that the actual easy axis is parallel to $\hat{\mathbf{b}}$.

\section{Magnon dispersion}

In this section, we calculate the spin-wave dispersion by means of the Holstein-Primakoff transformation [56,57]. Figure 7 shows the results obtained using the cRPA values

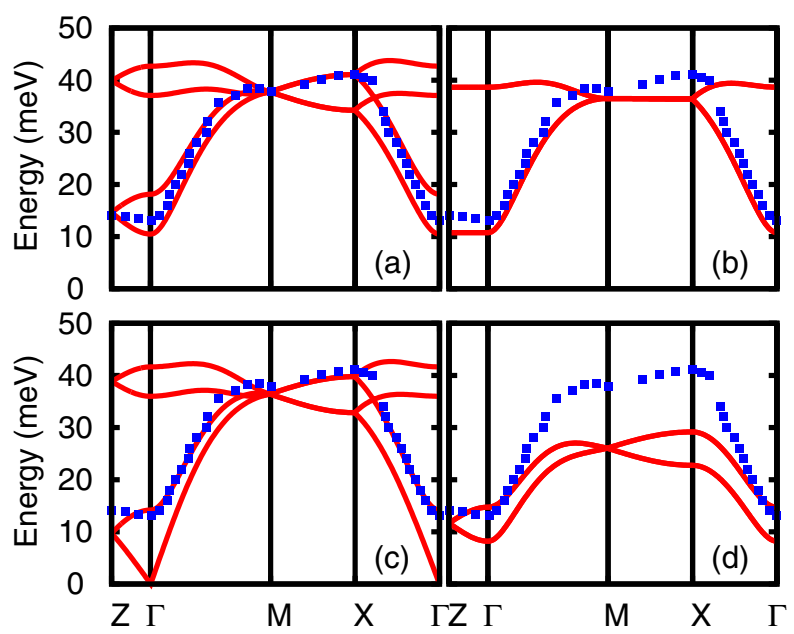

FIG. 7. Magnon dispersion of $\mathrm{Ca}_{2} \mathrm{RuO}_{4}$. Points: Experimental points showing the maximum of intensity taken from Fig. 3 in Ref. [33]. Lines: Spin-wave dispersion calculated by us with (a) all exchange and single-ion anisotropy parameters, (b) setting $\Gamma_{z}^{a}=\Gamma_{z}^{b}=0$, (c) setting $D_{a a}$ and $D_{b b}$ equal to the average value $\left(D_{a a}+D_{b b}\right) / 2$, and finally (d) setting to zero all single-ion anisotropy parameters but $D_{b b}$, redefined as $D_{b b}-D_{a a}$. All interactions have been calculated for the cRPA Coulomb parameters. 
of the Coulomb interaction and $\lambda_{\text {eff }} \sim 180 \mathrm{meV}$. As shown in panel (a), the main features of experimental data are well reproduced. The lower two branches split because of the interlayer magnetic couplings $\Gamma_{z}^{a}$ and $\Gamma_{z}^{b}$. This can be verified by comparing the results in panel (a) with those in panel (b); the latter were obtained by setting $\Gamma_{z}^{a}$ and $\Gamma_{z}^{b}$ to zero. The two upper branches are the result of band folding (the unit cell contains four formula units). Panel (d) shows results we obtained by setting all single-ion anisotropy terms to zero except from $D_{b b}$, redefined as $D_{b b}-D_{a a}$. Thus the superexchange couplings only contribute to about half the bandwidth, while the rest originates from single-ion anisotropy. Furthermore, the small in-plane anisotropy results in a finite excitation energy [33] at the $\Gamma$ point, larger than $10 \mathrm{meV}$. This can be seen in panel (c), where the orthorhombic anisotropy is set to zero. Similar conclusions can be achieved with cLDA parameters provided, however, that $\lambda_{\text {eff }} \sim 330 \mathrm{meV}$ and $D_{a a}-D_{b b} \sim 1 \mathrm{meV}$. The picture emerging from this analysis is thus different from the one suggested in Ref. [33] in which the magnon bandwidth is attributed almost completely to the exchange couplings.

\section{CONCLUSIONS}

In this work, we have studied the metal-insulator transition of $\mathrm{Ca}_{2} \mathrm{RuO}_{4}$ in the presence of spin-orbit interaction and anisotropic Coulomb interaction. We used the local-density approximation + dynamical mean-field theory (LDA+DMFT) approach. We use two sets of screened Coulomb parameters available in the literature, one calculated via the constrained random-phase approximation (CRPA) and the other via the constrained local-density approximation (cLDA). The LDA+DMFT results that we obtain with these two sets are qualitatively similar. Our calculations show that the change of structure from L-Pbca $\rightarrow \mathrm{S}$-Pbca is the most influential factor in determining the metal-insulator transition in $\mathrm{Ca}_{2} \mathrm{RuO}_{4}$, whereas spin-orbit coupling, magnetic ordering, and the anisotropic Coulomb interactions are not decisive. Furthermore, contrary to what static mean-field LDA+U calculations suggest [21], we find that the spin-orbit interaction slightly reduces the gap in the $x y$-orbitally-ordered insulating S-Pbca phase.

By means of many-body perturbation theory, we then compute the intersite magnetic couplings and the spin-orbitgenerated single-ion anisotropy tensor $D$. With this realistic set of parameters, we calculate the magnon dispersion via spinwave theory. The quantitative agreement with experiments is better when cRPA screened Coulomb parameters are used, but qualitatively the results are similar for both cRPA and cLDA parameter sets. We find that the $D$ tensor is key in determining the spin dynamics of $\mathrm{Ca}_{2} \mathrm{RuO}_{4}$ : it not only contributes to half the bandwidth, but, surprisingly, it also yields a sizable gap at the $\Gamma$ point via the small in-plane anisotropy $D_{a a}-D_{b b} \sim 1 \mathrm{meV}$.

\section{ACKNOWLEDGMENT}

We acknowledge financial support from the Deutsche Forschungsgemeinschaft through research unit FOR1346. Calculations were done on the Jülich Blue Gene/Q.

\section{APPENDIX}

Let us assume that the ground-state multiplet is the $S=1$ multiplet $\left|4 ; S, m_{S}\right\rangle$ with the electronic configuration $x y^{2} x z^{1} y z^{1}$ and energy $E(4)=2 \varepsilon_{\mathrm{CF}}+6 U-13 J$. The corresponding states can be found in Table III. In the $T \rightarrow 0$ limit, the diagonal elements of the atomic Green-function matrix are given by

$$
\begin{aligned}
G_{x y \sigma}(\omega)= & \frac{1}{3} \sum_{N=3} \sum_{m_{S}} \frac{\left|\left\langle 3\left|c_{x y \sigma}\right| 4 ; 1, m_{S}, x y\right\rangle\right|^{2}}{\omega-i \delta+\mu-E(4)+E(3)} \\
= & \frac{2 / 3}{\omega-i \delta+\mu-(3 U-4 J)} \\
& +\frac{1 / 3}{\omega-i \delta+\mu-(3 U-7 J)}
\end{aligned}
$$

Here $|N\rangle$ indicates all of the possible states with $N$ electrons,

$$
\begin{aligned}
G_{x z \sigma}(\omega)= & \frac{1}{3} \sum_{N=5} \sum_{m_{S}} \frac{\left|\left\langle 5\left|c_{x z \sigma}^{\dagger}\right| 4 ; 1, m_{S}, x y\right\rangle\right|^{2}}{\omega+i \delta+\mu+E(4)-E(5)} \\
& +\frac{1}{3} \sum_{N=3} \sum_{m_{S}} \frac{\left|\left\langle 3\left|c_{x z \sigma}\right| 4 ; 1, m_{S}, x y\right\rangle\right|^{2}}{\omega-i \delta+\mu-E(4)+E(3)} \\
= & \frac{1 / 4}{\omega-i \delta+\mu-\left(\varepsilon_{\mathrm{CF}}+3 U-7 J\right)} \\
& +\frac{1 / 4}{\omega-i \delta+\mu-\left(\varepsilon_{\mathrm{CF}}+3 U-9 J\right)} \\
& +\frac{1 / 2}{\omega+i \delta+\mu-\left(\varepsilon_{\mathrm{CF}}+4 U-7 J\right)} .
\end{aligned}
$$

These Green functions yield the correct Hartree-Fock highfrequency limit, $\Sigma_{x z}(\infty)-\Sigma_{x y}(\infty)=(U-5 J) / 2$. In the limit in which the spin-orbit interaction does not change (at least in a first approximation) the occupied state multiplet, we can neglect the effects of $\lambda_{x y}$. Thus, the most relevant effect for the Green function is that the following states gain/lose energy $\Delta E=\mp \sigma \lambda / 2$ :

$$
\begin{aligned}
& \left|5 ; \frac{1}{2}, \frac{\sigma}{2}\right\rangle_{ \pm}=\frac{i c_{y z \sigma} \pm c_{x z \sigma}}{\sqrt{2}}|6\rangle, \\
& \left|3 ; \frac{1}{2}, \frac{\sigma}{2}\right\rangle_{ \pm}=\frac{i\left|3 ; \frac{1}{2}, \frac{\sigma}{2}, y z\right\rangle_{\alpha} \pm\left|3 ; \frac{1}{2}, \frac{\sigma}{2}, x z\right\rangle_{\alpha}}{\sqrt{2}} .
\end{aligned}
$$

These states are only relevant for the $G_{x z / y z}$ Green function. Table III gives the list of all atomic multiplets for a $t_{2 g}$ atom with $N$ electrons in the absence of spin-orbit interactions. 
TABLE III. The atomic $t_{2 g}$ states $(m=x y, x z, y z)$ in the basis that diagonalize the Coulomb matrix. The label $\sigma$ in the first column takes the value \pm 1 , while in the states it means $\uparrow$ or $\downarrow$. The labels $m, m^{\prime}$, and $m^{\prime \prime}$ indicate different orbitals.

\begin{tabular}{|c|c|c|c|c|c|}
\hline \multicolumn{3}{|l|}{$\left|N ; S, m_{S}\right\rangle$} & \multicolumn{2}{|l|}{$E(N)$} & \\
\hline$|0\rangle$ & & & 0 & & \\
\hline$\left|1 ; \frac{1}{2}, \frac{\sigma}{2}\right\rangle$ & $=$ & $c_{m \sigma}^{\dagger}|0\rangle$ & $\varepsilon_{m}$ & & \\
\hline$|2 ; 0,0\rangle_{a}$ & $=$ & $\frac{1}{\sqrt{3}}\left[c_{x z \uparrow}^{\dagger} c_{x z \downarrow}^{\dagger}+c_{y z \uparrow}^{\dagger} c_{y z \downarrow}^{\dagger}+c_{x y \uparrow}^{\dagger} c_{x y \downarrow}^{\dagger}\right]|0\rangle$ & $\frac{4}{3} \varepsilon_{\mathrm{CF}}$ & $+U$ & $+2 J$ \\
\hline$|2 ; 0,0\rangle_{b}$ & $=$ & $\frac{1}{\sqrt{6}}\left[c_{x z \uparrow}^{\dagger} c_{x z \downarrow}^{\dagger}+c_{y z \uparrow}^{\dagger} c_{y z \downarrow}^{\dagger}-2 c_{x y \uparrow}^{\dagger} c_{x y \downarrow}^{\dagger}\right]|0\rangle$ & $\frac{2}{3} \varepsilon_{\mathrm{CF}}$ & $+U$ & $-J$ \\
\hline$|2 ; 0,0\rangle_{c}$ & $=$ & $\frac{1}{\sqrt{2}}\left[c_{x z \uparrow}^{\dagger} c_{x z \downarrow}^{\dagger}-c_{y z \uparrow}^{\dagger} c_{y z \downarrow}^{\dagger}\right]|0\rangle$ & $2 \varepsilon_{\mathrm{CF}}$ & $+U$ & $-J$ \\
\hline$\left|2 ; 1, \sigma, m^{\prime \prime}\right\rangle$ & $=$ & $c_{m \sigma}^{\dagger} c_{m^{\prime} \sigma}^{\dagger}|0\rangle$ & $\varepsilon_{m}+\varepsilon_{m^{\prime}}$ & $+U$ & $-3 J$ \\
\hline$\left|2 ; 1,0, m^{\prime \prime}\right\rangle$ & $=$ & $\frac{1}{\sqrt{2}}\left[c_{m \uparrow}^{\dagger} c_{m^{\prime} \downarrow}^{\dagger}+c_{m \downarrow}^{\dagger} c_{m^{\prime} \uparrow}^{\dagger}\right]|0\rangle$ & $\varepsilon_{m}+\varepsilon_{m^{\prime}}$ & $+U$ & $-3 J$ \\
\hline$\left|2 ; 0,0, m^{\prime \prime}\right\rangle$ & $=$ & $\frac{1}{\sqrt{2}}\left[c_{m \uparrow}^{\dagger} c_{m^{\prime} \downarrow}^{\dagger}-c_{m \downarrow}^{\dagger} c_{m^{\prime} \uparrow}^{\dagger}\right]|0\rangle$ & $\varepsilon_{m}+\varepsilon_{m^{\prime}}$ & $+U$ & $-J$ \\
\hline$\left|3 ; \frac{3}{2}, \frac{3 \sigma}{2}\right\rangle$ & $=$ & $c_{x z \sigma}^{\dagger} c_{y z \sigma}^{\dagger} c_{x y \sigma}^{\dagger}|0\rangle$ & $2 \varepsilon_{\mathrm{CF}}$ & $+3 U$ & $-9 J$ \\
\hline$\left|3 ; \frac{3}{2}, \frac{\sigma}{2}\right\rangle$ & $=$ & $\frac{1}{\sqrt{3}}\left[c_{x z \sigma}^{\dagger} c_{y z \sigma}^{\dagger} c_{x y-\sigma}^{\dagger}+c_{x z \sigma}^{\dagger} c_{y z-\sigma}^{\dagger} c_{x y \sigma}^{\dagger}+c_{x z-\sigma}^{\dagger} c_{y z \sigma}^{\dagger} c_{x y \sigma}^{\dagger}\right]|0\rangle$ & $2 \varepsilon_{\mathrm{CF}}$ & $+3 U$ & $-9 J$ \\
\hline$\left|3 ; \frac{1}{2}, \frac{\sigma}{2}\right\rangle_{a}$ & $=$ & $\frac{1}{\sqrt{6}}\left[-2 c_{x z \sigma}^{\dagger} c_{y z \sigma}^{\dagger} c_{x y-\sigma}^{\dagger}+c_{x z \sigma}^{\dagger} c_{y z-\sigma}^{\dagger} c_{x y \sigma}^{\dagger}+c_{x z-\sigma}^{\dagger} c_{y z \sigma}^{\dagger} c_{x y \sigma}^{\dagger}\right]|0\rangle$ & $2 \varepsilon_{\mathrm{CF}}$ & $+3 U$ & $-6 J$ \\
\hline$\left|3 ; \frac{1}{2}, \frac{\sigma}{2}\right\rangle_{b}$ & $=$ & $\frac{1}{\sqrt{2}}\left[c_{x z \sigma}^{\dagger} c_{y z-\sigma}^{\dagger}-c_{x z-\sigma}^{\dagger} c_{y z \sigma}^{\dagger}\right] c_{x y \sigma}^{\dagger}|0\rangle$ & $2 \varepsilon_{\mathrm{CF}}$ & $+3 U$ & $-6 J$ \\
\hline$\left|3 ; \frac{1}{2}, \frac{\sigma}{2}, m\right\rangle_{a}$ & $=$ & $\frac{1}{\sqrt{2}}\left[c_{m^{\prime} \uparrow}^{\dagger} c_{m^{\prime} \downarrow}^{\dagger}+c_{m^{\prime \prime} \uparrow}^{\dagger} c_{m^{\prime \prime} \downarrow}^{\dagger}\right] c_{m \sigma}^{\dagger}|0\rangle$ & $\varepsilon_{m}+\varepsilon_{m^{\prime}}+\varepsilon_{m^{\prime \prime}}$ & $+3 U$ & $-4 J$ \\
\hline$\left|3 ; \frac{1}{2}, \frac{\sigma}{2}, m\right\rangle_{b}$ & $=$ & $\frac{1}{\sqrt{2}}\left[c_{m^{\prime} \uparrow}^{\dagger} c_{m^{\prime} \downarrow}^{\dagger}-c_{m^{\prime \prime} \uparrow}^{\dagger} c_{m^{\prime \prime} \downarrow}^{\dagger}\right] c_{m \sigma}^{\dagger}|0\rangle$ & $\varepsilon_{m}+\varepsilon_{m^{\prime}}+\varepsilon_{m^{\prime \prime}}$ & $+3 U$ & $-6 J$ \\
\hline$\left|4 ; 1, \sigma, m^{\prime \prime}\right\rangle$ & $=$ & $c_{m \sigma}^{\dagger} c_{m^{\prime} \sigma}^{\dagger} c_{m^{\prime \prime} \uparrow}^{\dagger} c_{m^{\prime \prime} \downarrow}^{\dagger}|0\rangle$ & $4 \varepsilon_{\mathrm{CF}}-\varepsilon_{m}-\varepsilon_{m^{\prime}}$ & $+6 U$ & $-13 J$ \\
\hline$\left|4 ; 1,0, m^{\prime \prime}\right\rangle$ & $=$ & $\frac{1}{\sqrt{2}}\left[c_{m \uparrow}^{\dagger} c_{m^{\prime} \downarrow}^{\dagger}+c_{m \downarrow}^{\dagger} c_{m^{\prime} \uparrow}^{\dagger}\right] c_{m^{\prime \prime} \uparrow}^{\dagger} c_{m^{\prime \prime} \downarrow}^{\dagger}|0\rangle$ & $4 \varepsilon_{\mathrm{CF}}-\varepsilon_{m}-\varepsilon_{m^{\prime}}$ & $+6 U$ & $-13 J$ \\
\hline$\left|4 ; 0,0, m^{\prime \prime}\right\rangle$ & $=$ & $\frac{1}{\sqrt{2}}\left[c_{m \uparrow}^{\dagger} c_{m^{\prime} \downarrow}^{\dagger}-c_{m \downarrow}^{\dagger} c_{m^{\prime} \uparrow}^{\dagger}\right] c_{m^{\prime \prime} \uparrow}^{\dagger} c_{m^{\prime \prime} \downarrow}^{\dagger}|0\rangle$ & $4 \varepsilon_{\mathrm{CF}}-\varepsilon_{m}-\varepsilon_{m^{\prime}}$ & $+6 U$ & $-11 J$ \\
\hline$|4 ; 0,0\rangle_{a}$ & $=$ & $\frac{1}{\sqrt{3}}\left[c_{x z \uparrow}^{\dagger} c_{x z \downarrow}^{\dagger} c_{y z \uparrow}^{\dagger} c_{y z \downarrow}^{\dagger}+c_{y z \uparrow}^{\dagger} c_{y z \downarrow}^{\dagger} c_{x y \uparrow}^{\dagger} c_{x y \downarrow}^{\dagger}+c_{x y \uparrow}^{\dagger} c_{x y \downarrow}^{\dagger} c_{x z \uparrow}^{\dagger} c_{x z \downarrow}^{\dagger}\right]|0\rangle$ & $4 \varepsilon_{\mathrm{CF}}-\frac{4}{3} \varepsilon_{\mathrm{CF}}$ & $+6 U$ & $-8 J$ \\
\hline$|4 ; 0,0\rangle_{b}$ & $=$ & $\frac{1}{\sqrt{6}}\left[c_{x z \uparrow}^{\dagger} c_{x z \downarrow}^{\dagger} c_{y z \uparrow}^{\dagger} c_{y z \downarrow}^{\dagger}+c_{y z \uparrow}^{\dagger} c_{y z \downarrow}^{\dagger} c_{x y \uparrow}^{\dagger} c_{x y \downarrow}^{\dagger}-2 c_{x y \uparrow}^{\dagger} c_{x y \downarrow}^{\dagger} c_{x z \uparrow}^{\dagger} c_{x z \downarrow}^{\dagger}\right]|0\rangle$ & $4 \varepsilon_{\mathrm{CF}}-\frac{2}{3} \varepsilon_{\mathrm{CF}}$ & $+6 U$ & $-11 J$ \\
\hline$|4 ; 0,0\rangle_{c}$ & $=$ & $\frac{1}{\sqrt{2}}\left[c_{x z \uparrow}^{\dagger} c_{x z \downarrow}^{\dagger} c_{y z \uparrow}^{\dagger} c_{y z \downarrow}^{\dagger}-c_{y z \uparrow}^{\dagger} c_{y z \downarrow}^{\dagger} c_{x y \uparrow}^{\dagger} c_{x y \downarrow}^{\dagger}\right]|0\rangle$ & $2 \varepsilon_{\mathrm{CF}}$ & $+6 U$ & $-11 J$ \\
\hline$\left|5 ; \frac{1}{2}, \frac{\sigma}{2}\right\rangle$ & $=$ & $c_{m \sigma}^{\dagger} c_{m^{\prime} \uparrow}^{\dagger} c_{m^{\prime} \downarrow}^{\dagger} c_{m^{\prime \prime} \uparrow}^{\dagger} c_{m^{\prime \prime} \downarrow}^{\dagger}|0\rangle$ & $4 \varepsilon_{\mathrm{CF}}-\varepsilon_{m}$ & $+10 U$ & $-20 J$ \\
\hline$|6\rangle$ & $=$ & $c_{x z \uparrow}^{\dagger} c_{x z \downarrow}^{\dagger} c_{y z \uparrow}^{\dagger} c_{y z \downarrow}^{\dagger} c_{x y \uparrow}^{\dagger} c_{x y \downarrow}^{\dagger}|0\rangle$ & $4 \varepsilon_{\mathrm{CF}}$ & $+15 U$ & $-30 J$ \\
\hline
\end{tabular}

[1] K. Ishida, H. Mukuda, Y. Kitaoka, K. Asayama, Z. Q. Mao, Y. Mori, and Y. Maeno, Nature (London) 396, 658 (1998).

[2] L. de'Medici, J. Mravlje, and A. Georges, Phys. Rev. Lett. 107, 256401 (2011).

[3] D. Sutter et al., arXiv:1610.02854. In this work, $J$ is estimated as $\sim 0.4 \mathrm{eV}$, i.e., close to the cRPA value. By direct comparison of our spectral functions with the experimental data, $J=0.7 \mathrm{eV}$ (cLDA value) apparently cannot be fully ruled out, however.

[4] J. S. Lee, Y. S. Lee, T. W. Noh, S.-J. Oh, J. Yu, S. Nakatsuji, H. Fukazawa, and Y. Maeno, Phys. Rev. Lett. 89, 257402 (2002); S. Nakatsuji, D. Hall, L. Balicas, Z. Fisk, K. Sugahara, M. Yoshioka, and Y. Maeno, ibid. 90, 137202 (2003).

[5] J. P. Carlo, T. Goko, I. M. Gat-Malureanu, P. L. Russo, A. T. Savici, A. A. Aczel, G. J. MacDougall, J. A. Rodriguez, T. J. Williams, G. M. Luke, C. R. Wiebe, Y. Yoshida, S. Nakatsuji, Y. Maeno, T. Taniguchi, and Y. J. Uemura, Nat. Mater. 11, 323 (2012).

[6] C. S. Alexander, G. Cao, V. Dobrosavljevic, S. McCall, J. E. Crow, E. Lochner, and R. P. Guertin, Phys. Rev. B 60, R8422(R) (1999).

[7] S. Nakatsuji and Y. Maeno, Phys. Rev. Lett. 84, 2666 (2000).
[8] O. Friedt, M. Braden, G. André, P. Adelmann, S. Nakatsuji, and Y. Maeno, Phys. Rev. B 63, 174432 (2001).

[9] M. Braden, G. André, S. Nakatsuji, and Y. Maeno, Phys. Rev. B 58, 847 (1998).

[10] F. Nakamura, T. Goko, M. Ito, T. Fujita, S. Nakatsuji, H. Fukazawa, Y. Maeno, P. Alireza, D. Forsythe, and S. R. Julian, Phys. Rev. B 65, 220402(R) (2002).

[11] P. Steffens, O. Friedt, P. Alireza, W. G. Marshall, W. Schmidt, F. Nakamura, S. Nakatsuji, Y. Maeno, R. Lengsdorf, M. M. Abd-Elmeguid, and M. Braden, Phys. Rev. B 72, 094104 (2005).

[12] G. Cao, S. McCall, V. Dobrosavljevic, C. S. Alexander, J. E. Crow, and R. P. Guertin, Phys. Rev. B 61, R5053(R) (2000).

[13] M. Neupane, P. Richard, Z.-H. Pan, Y.-M. Xu, R. Jin, D. Mandrus, X. Dai, Z. Fang, Z. Wang, and H. Ding, Phys. Rev. Lett. 103, 097001 (2009).

[14] A. Shimoyamada, K. Ishizaka, S. Tsuda, S. Nakatsuji, Y. Maeno, and S. Shin, Phys. Rev. Lett. 102, 086401 (2009).

[15] I. Zegkinoglou, J. Strempfer, C. S. Nelson, J. P. Hill, J. Chakhalian, C. Bernhard, J. C. Lang, G. Srajer, H. Fukazawa, S. Nakatsuji, Y. Maeno, and B. Keimer, Phys. Rev. Lett. 95, 136401 (2005).

[16] T. Hotta and E. Dagotto, Phys. Rev. Lett. 88, 017201 (2001). 
[17] A. Liebsch and H. Ishida, Phys. Rev. Lett. 98, 216403 (2007).

[18] C. Knecht, N. Blümer, and P. G. J. van Dongen, Phys. Rev. B 72, 081103(R) (2005).

[19] S. Okamoto and A. J. Millis, Phys. Rev. B 70, 195120 (2004).

[20] J. H. Jung, Z. Fang, J. P. He, Y. Kaneko, Y. Okimoto, and Y. Tokura, Phys. Rev. Lett. 91, 056403 (2003).

[21] G.-Q. Liu, Phys. Rev. B 88, 104428 (2013).

[22] G.-Q. Liu, Phys. Rev. B 84, 235136 (2011).

[23] E. Gorelov, M. Karolak, T. O. Wehling, F. Lechermann, A. I. Lichtenstein, and E. Pavarini, Phys. Rev. Lett. 104, 226401 (2010).

[24] V. I. Anisimov, I. A. Nekrasov, D. E. Kondakov, T. M. Rice, and M. Sigrist, Eur. Phys. J. B 25, 191 (2002).

[25] E. Ko, B. J. Kim, C. Kim, and H. J. Choi, Phys. Rev. Lett. 98, 226401 (2007).

[26] N. Arakawa and M. Ogata, Phys. Rev. B 86, 125126 (2012).

[27] E. Pavarini, S. Biermann, A. Poteryaev, A. I. Lichtenstein, A. Georges, and O. K. Andersen, Phys. Rev. Lett. 92, 176403 (2004); E. Pavarini, A. Yamasaki, J. Nuss, and O. K. Andersen, New J. Phys. 7, 188 (2005).

[28] E. Pavarini and I. I. Mazin, Phys. Rev. B 74, 035115 (2006).

[29] M. W. Haverkort, I. S. Elfimov, L. H. Tjeng, G. A. Sawatzky, and A. Damascelli, Phys. Rev. Lett. 101, 026406 (2008).

[30] G. Zhang, E. Gorelov, E. Sarvestani, and E. Pavarini, Phys. Rev. Lett. 116, 106402 (2016).

[31] C. G. Fatuzzo, M. Dantz, S. Fatale, P. Olalde-Velasco, N. E. Shaik, B. Dalla Piazza, S. Toth, J. Pelliciari, R. Fittipaldi, A. Vecchione, N. Kikugawa, J. S. Brooks, H. M. Rønnow, M. Grioni, Ch. Rüegg, T. Schmitt, and J. Chang, Phys. Rev. B 91, 155104 (2015).

[32] M. Behrmann, C. Piefke, and F. Lechermann, Phys. Rev. B 86, 045130 (2012).

[33] S. Kunkemöller, D. Khomskii, P. Steffens, A. Piovano, A. A. Nugroho, and M. Braden, Phys. Rev. Lett. 115, 247201 (2015).

[34] A. Jain, M. Krautloher, J. Porras, G. H. Ryu, D. P. Chen, D. L. Abernathy, J. T. Park, A. Ivanov, J. Chaloupka, G. Khaliullin, B. Keimer, and B. J. Kim, arXiv:1510.07011.

[35] A. Akbari and G. Khaliullin, Phys. Rev. B 90, 035137 (2014).

[36] G. Khaliullin, Phys. Rev. Lett. 111, 197201 (2013).

[37] We performed the LDA calculations for the crystal structure reported in Refs. [8,9]. For the muffin-tin radii, we used 2.15 (Ca), $1.96(\mathrm{Ru})$, and $1.74(\mathrm{O})$ for S-Pbca structures and slightly smaller for the L-Pbca structure. Self-consistent calculations were performed using a mesh of $11 \times 11 \times 4 k$-points.

[38] P. Blaha, K. Schwarz, G. Madsen, D. Kvasnicka and J. Luitz, WIEN2K, An Augmented Plane Wave + Local Orbitals Program for Calculating Crystal Properties (Technische Universität Wien, Austria, 2001).

[39] A. A. Mostofi, J. R. Yates, Y.-S. Lee, I. Souza, D. Vanderbilt, and N. Marzari, Comput. Phys. Commun. 178, 685 (2008); J. Kuneš, R. Arita, P. Wissgott, A. Toschi, H. Ikeda, and K. Held, ibid. 181, 1888 (2010).

[40] For a pedagogical presentation, see, e.g., E. Pavarini, The $L D A+D M F T$ Approach, in The LDA+DMFT Approach to Strongly Correlated Materials, edited by E. Pavarini, E. Koch, D. Vollhardt, and A. Lichtenstein, Modeling and Simulation Vol. 1 (Forschungszentrum Jülich, Jülich, 2011), Link: http://www. cond-mat.de/events/correl11/manuscripts/pavarini.pdf.
[41] Z. V. Pchelkina, I. A. Nekrasov, Th. Pruschke, A. Sekiyama, S. Suga, V. I. Anisimov, and D. Vollhardt, Phys. Rev. B 75, 035122 (2007).

[42] J. Mravlje, M. Aichhorn, T. Miyake, K. Haule, G. Kotliar, and A. Georges, Phys. Rev. Lett. 106, 096401 (2011).

[43] M. Schmidt, T. R. Cummins, M. Bürk, D. H. Lu, N. Nücker, S. Schuppler, and F. Lichtenberg, Phys. Rev. B 53, R14761(R) (1996).

[44] T. Yokoya, A. Chainani, T. Takahashi, H. Katayama-Yoshida, M. Kasai, Y. Tokura, N. Shanthi, and D. D. Sarma, Phys. Rev. B 53, 8151 (1996).

[45] E. Z. Kurmaev, S. Stadler, D. L. Ederer, Y. Harada, S. Shin, M. M. Grush, T. A. Callcott, R. C. C. Perera, D. A. Zatsepin, N. Ovechkina, M. Kasai, Y. Tokura, T. Takahashi, K. Chandrasekaran, R. Vijayaraghavan, and U. V. Varadaraju, Phys. Rev. B 57, 1558 (1998).

[46] For isotropic Coulomb interactions, $H_{\mathrm{dc}}$ is a mere shift of the chemical potential. For calculations with anisotropic Coulomb interactions, we use the around mean-field-like form of $H_{\mathrm{dc}}$ because the LDA occupations for $t_{2 g}$ orbitals are quite close, e.g., for the S-Pbca structure they take the values $n_{x y \sigma}=0.785$, $n_{y z \sigma}=0.606$, and $n_{x z \sigma}=0.609$.

[47] A. Flesch, E. Gorelov, E. Koch, and E. Pavarini, Phys. Rev. B 87, 195141 (2013).

[48] E. Gull, A. J. Millis, A. I. Lichtenstein, A. N. Rubtsov, M. Troyer, and P. Werner, Rev. Mod. Phys. 83, 349 (2011).

[49] A. N. Rubtsov, V. V. Savkin, and A. I. Lichtenstein, Phys. Rev. B 72, 035122 (2005).

[50] For a pedagogical introduction, see M. Jarrell, The Maximum Entropy Method, in Correlated Electrons: From Models to Materials Modeling and Simulation, edited by E. Pavarini, E. Koch, F. Anders, and M. Jarrell (Verlag des Forschungszentrum, Jülich, 2012), Vol. 2.

[51] This is a consequence of the static mean-field approximation adopted in LDA $+\mathrm{U}$ for the Coulomb interaction. Indeed, the same effect is obtained by solving the Hubbard model for the SPbca structure with Hartree-Fock; this is true both for a $t_{2 g}$-only model and one additionally including the empty $e_{g}$ states.

[52] The spread $\left\langle\left|\mathbf{r}^{2}\right|\right\rangle$ is $4.91 \AA^{2}$ for the $x y$ orbital, $3.77 \AA^{2}$ for the $y z$ orbital, and $3.68 \AA^{2}$ for the $y z$ orbital.

[53] The atomic $t_{2 g}$ multiplet $\left|j_{\text {tot }}\right\rangle$ with $j_{\text {tot }}=0$ can be written as follows:

$$
\begin{aligned}
|0\rangle= & \frac{1}{\sqrt{3}}\left[-c_{1 \uparrow}^{\dagger} c_{1 \downarrow}^{\dagger} c_{-1 \uparrow}^{\dagger} c_{x y \uparrow}^{\dagger}|0\rangle+c_{1 \downarrow}^{\dagger} c_{-1 \uparrow}^{\dagger} c_{-1 \downarrow}^{\dagger} c_{x y \downarrow}^{\dagger}|0\rangle\right. \\
& \left.-\frac{1}{\sqrt{2}}\left(c_{1 \uparrow}^{\dagger} c_{-1 \downarrow}^{\dagger}+c_{1 \downarrow}^{\dagger} c_{-1 \uparrow}^{\dagger}\right) c_{x y \uparrow}^{\dagger} c_{x y \downarrow}^{\dagger}|0\rangle\right]
\end{aligned}
$$

where \pm 1 are $d$ spherical harmonics with $m= \pm 1$. When this is the ground state, the orbital polarization is zero.

[54] In Ref. [21], a density-density form of the Coulomb Hamiltonian interaction is used, however in the basis of the $|m= \pm 1\rangle$ spherical harmonics, i.e., the states $|3 / 2\rangle=|-1\rangle_{\uparrow},|1 / 2\rangle=$ $|-1\rangle_{\downarrow},|-1 / 2\rangle=|+1\rangle_{\uparrow}$, and $|-3 / 2\rangle=|+1\rangle_{\downarrow}$. By rotating the Coulomb interaction for the $x z, y z$ states to that basis, however, we find the following Coulomb term (see, e.g., Ref. [40] for a pedagogical derivation):

$$
\begin{aligned}
H_{U}= & (U-J)\left(n_{\frac{3}{2}}+n_{-\frac{1}{2}}\right)\left(n_{\frac{1}{2}}+n_{-\frac{3}{2}}\right)+(U-3 J) n_{\frac{3}{2}} n_{-\frac{1}{2}} \\
& +(U-3 J) n_{-\frac{3}{2}} n_{\frac{1}{2}}-2 J\left(c_{\frac{3}{2}}^{\dagger} c_{\frac{1}{2}} c_{-\frac{3}{2}}^{\dagger} c_{-\frac{1}{2}}+c_{\frac{1}{2}}^{\dagger} c_{\frac{3}{2}} c_{-\frac{1}{2}}^{\dagger} c_{-\frac{3}{2}}\right) .
\end{aligned}
$$


The prefactor of the pair-hopping term is zero. This Hamiltonian differs from the classical density-density form (even after neglecting the spin-flip term). For the Hamiltonian above, the mean-field enhancement of the spin-orbit coupling obtained from $H_{U}$ is, as expected, identical to the one we obtain in LDA+DMFT in the large frequency limit.
[55] G. Cao, S. McCall, M. Shepard, J. E. Crow, and R. P. Guertin, Phys. Rev. B 56, R2916(R) (1997).

[56] M. P. H. Thurlings, E. Frikkee, and H. W. de Wijn, Phys. Rev. B 25, 4750 (1982).

[57] F. Lu and X. Dai, Chin. Phys. B 21, 027502 (2012). 